\title{
Association of functional and structural social support with chronic kidney disease among African Americans: the Jackson Heart Study
}

Rasheeda K. Hall ${ }^{1 *}$ (D), Clemontina A. Davenport ${ }^{1}$, Mario Sims², Cathleen Colón-Emeric ${ }^{1}$, Tiffany Washington ${ }^{3}$, Jennifer St. Clair Russell', Jane Pendergast ${ }^{1}$, Nrupen Bhavsarr, Julia Scialla', Crystal C. Tyson? ${ }^{1}$, Wei Wang ${ }^{2}$, Yuan-I Min², Bessie Young ${ }^{4}$, L. Ebony Boulware ${ }^{1}$ and Clarissa J. Diamantidis ${ }^{1}$

\begin{abstract}
Background: There is limited evidence on the relationship between social support and renal outcomes in African Americans. We sought to determine the association of social support with prevalent chronic kidney disease (CKD) and kidney function decline in an African American cohort. We also examined whether age modifies the association between social support and kidney function decline.

Methods: We identified Jackson Heart Study (JHS) participants with baseline (Exam in 2000-2004) functional and structural social support data via the Interpersonal Support Evaluation List (ISEL) and social network size questions, respectively. With ISEL as our primary exposure variable, we performed multivariable regression models to evaluate the association between social support and prevalent CKD [estimated glomerular filtration rate (eGFR) $<60 \mathrm{ml} / \mathrm{min} / 1.73 \mathrm{~m} 2$ or urine albumin-creatinine ratio (ACR) $\geq 30 \mathrm{mg} / \mathrm{g}$ ], eGFR decline, and rapid renal function decline (RRFD) (>30\% decrease in eGFR over approximately 10 years). All models were adjusted for baseline sociodemographics, diabetes, hypertension, smoking status, and body mass index; models for eGFR decline and RRFD were additionally adjusted for eGFR and ACR. In models for eGFR decline, we assessed for interaction between age and social support. For secondary analyses, we replaced ISEL with its individual domains (appraisal, belonging, self-esteem, and tangible) and social network size in separate models as exposure variables.
\end{abstract}

Results: Of 5301 JHS participants, 4015 (76\%) completed the ISEL at baseline. 843 (21\%) had low functional social support (ISEL score <32). Participants with low (vs. higher) functional social support were more likely to have lower income (47\% vs. $28 \%$ ), be current or former tobacco users (39\% vs. $30 \%)$, have diabetes (25\% vs. $21 \%$ ) or CKD (14\% vs. 12\%). After multivariable adjustment, neither ISEL or social network size were independently associated with prevalent CKD, eGFR decline, or RRFD. Of the ISEL domains, only higher self-esteem was associated with lower odds of prevalent CKD [OR 0.94 (95\% Cl 0.89-0.99)]. The associations between social support measures and eGFR decline were not modified by age.

Conclusions: In this African-American cohort, social support was not associated with prevalent CKD or kidney function decline. Further inquiry of self-esteem's role in CKD self-management and renal outcomes is warranted.

Keywords: Minority health, Social network, Chronic renal insufficiency, Aged

\footnotetext{
* Correspondence: rasheeda.stephens@duke.edu

'Duke University, Durham, NC, USA

Full list of author information is available at the end of the article
}

(c) The Author(s). 2019 Open Access This article is distributed under the terms of the Creative Commons Attribution 4.0 International License (http://creativecommons.org/licenses/by/4.0/), which permits unrestricted use, distribution, and reproduction in any medium, provided you give appropriate credit to the original author(s) and the source, provide a link to the Creative Commons license, and indicate if changes were made. The Creative Commons Public Domain Dedication waiver (http://creativecommons.org/publicdomain/zero/1.0/) applies to the data made available in this article, unless otherwise stated. 


\section{Background}

African Americans bear a disproportionate burden of chronic kidney disease (CKD) [1]. Factors that contribute to this health disparity include sociodemographics, quality or access to care, and lifestyle, while nearly half of this excess risk is unknown [2]. One related psychosocial factor that may influence this excess risk is social support [3]. Social support refers to perceived emotional, material, or informational resources provided by others and/or size of one's social network [4]. The presence of adequate social support is associated with lower risk of morbidity and mortality in the general population [5-11]. This lower risk is explained by social support's facilitation of health-promoting behaviors [12]. In African Americans, social support buffers against the long-term health effects of stress, including stressful experiences of perceived discrimination [13], in addition to its association with improved glycemic control and blood pressure in this population [14, 15]. Thus evidence suggests that social support may have a meaningful association with CKD outcomes in African Americans.

Social support influences chronic disease self-management via both functional and structural pathways [11]. Functional social support is based on the stress-buffering hypothesis, and refers to specific resources provided by a person's social network (e.g., emotional support, socialization, financial assistance, advice) that can help them cope with life stress and gain confidence in managing their chronic disease, which positively impacts physiological processes $[12,16]$. Structural social support, based on social network theory that isolation negatively impacts health, refers to the size of a person's social network (i.e., number of people with whom one has meaningful and frequent contact) available to provide functional social support [5]. Prior studies in African Americans show both functional and structural social support are associated with both improved health outcomes and self-management behaviors [14, 15, 17, 18]. In a cohort of African Americans with hypertensive CKD, social support was significantly higher among those who reported better quality of life [19]. However, it remains unknown whether functional or structural social support are individually associated with CKD outcomes.

The objective of this study is to determine the association of both functional and structural social support with prevalent CKD and kidney function decline using data from the Jackson Heart Study (JHS), an all African American cohort study. Our hypothesis is that low social support would be associated with prevalent CKD and greater kidney function decline. Because there is a larger burden of disability, cognitive impairment, and social isolation among older adults [20], older adults may be more vulnerable to the effects of social support on selfmanagement behaviors that mitigate risk of kidney function decline [21, 22]. Therefore, we also examined the extent to which age modifies the association of social support with kidney function decline.

\section{Methods \\ Study design and population}

We conducted an observational study of social support and kidney function outcomes in African Americans using data from JHS participants. The JHS is a communitybased cohort study designed to evaluate heart disease risk factors in African Americans residing in the tri-county area (Hinds, Rankin, and Madison) of Jackson, Mississippi [23-25]. Briefly, 5301 African Americans ages 21 to 94 years old enrolled and participated in an initial study evaluation between 2000 and 2004 (Exam 1) and subsequent follow-up evaluations between 2005 and 2008 (Exam 2) and 2009-2013 (Exam 3). The JHS protocol was approved by the Institutional Review Boards at Mississippi Medical Center, Jackson State University, and Tougaloo College, and all participants provided informed consent.

For these analyses, we included JHS participants who completed social support measures and laboratory tests of kidney function at Exam 1. Those missing these data were excluded. For analyses of kidney function decline, we limited the cohort to JHS participants with diabetes, hypertension and/or CKD at Exam 1 and excluded JHS participants who reported a history of dialysis or kidney transplant at any visit (to avoid uncertainty about the accuracy of reported eGFR values).

\section{Primary independent variable: social support}

Our primary exposure of interest was functional social support, the nature by which interpersonal relationships provide support, assessed using the Interpersonal Support Evaluation List (ISEL) instrument. The ISEL is a validated self-administered instrument comprised of Likert-type items that represent four domains of functional social support, which include: 1) appraisal, 2) belonging, 3) selfesteem, and 4) tangible [26, 27]. The appraisal domain measures perceived availability of a confidant to talk to about one's problems. The belonging domain measures perceived availability of people with whom one can do things. The self-esteem domain measures the extent to which someone views themselves positively when comparing one's self with others. The tangible domain measures perceived availability of material aid, such as financial or transportation assistance. Each of the 4 domains contributes 12 points. Using the 16-item ISEL instrument, we used a standardized approach to compute summed ISEL scores (range 0 to 48) [27]. When used as a dichotomous variable, an ISEL score $<32$ was used to indicate low functional social support [8].

We secondarily assessed structural social support, often assessed by social network size beyond immediate 
family members. Developed for this study, we assessed social network size from three items adapted from the previously validated Berkman Social Network Index (Cronbach's $\alpha$ from our analytic dataset was 0.73 ) [5, 6]: 1) "how many close friends do you have?", 2) "how many close relatives do you have?", and 3) "how many of these friends or relatives do you see at least once a month?". Possible responses for each question were: "none", " 1 or 2 ", "3 to 5", "6 to 9", or "10 or more". We designated a score to each item ranging from 0 to 4 , respectively. We then computed an overall social network size as a sum of all three items, ranging from 0 to 12 to quantify structural social support. Aligning with a two-thirds cutoff to indicate low social support using the ISEL, social network size $<8$ was used to categorize participants as having low structural social support. Both the ISEL and social network size questions were provided to JHS participants during the initial home interview.

\section{Covariates}

We identified the following potential confounders of the relationship between social support and kidney function outcomes: 1) demographics (age, sex), 2) socioeconomic status (education [defined as $\leq$ high school degree vs. $>$ high school degree], income [defined as income $\leq 1.5$ times the poverty level vs. $>1.5$ times the poverty level and an additional category for missing income level]), 3) diabetes (defined as fasting glucose $\geq 126 \mathrm{mg} / \mathrm{dL}$ or $\mathrm{HbA} 1 \mathrm{c} \geq 6.5 \%$ or actual use of diabetic medications), 4) hypertension (defined as blood pressure [BP] $>140 / 90$ or self-report use of BP lowering meds), 5) smoking status (active/former vs. never-smoker based on self-report), and 6) body mass index (BMI) defined as $\mathrm{kg} / \mathrm{m}^{2}$.

\section{Primary dependent variable: kidney function}

The outcomes were 1) prevalent CKD and 2) estimated glomerular filtration rate (eGFR) decline over follow-up. We defined prevalent CKD as an eGFR $<60 \mathrm{ml} / \mathrm{min} /$ $1.73 \mathrm{~m}^{2}$ using the Chronic Kidney Disease-Epidemiology Collaboration (CKD-EPI) estimating equation ${ }^{11}$ or a urine albumin-to-creatinine ratio (ACR) $\geq 30 \mathrm{mg} / \mathrm{g}$ at Exam 1. We defined decline in eGFR as the annualized rate of change in eGFR $\left(\mathrm{ml} / \mathrm{min} / 1.73 \mathrm{~m}^{2}\right)$ from Exam 1 to Exam 3; based on the following equation: 365.25 days $\times\left(\mathrm{eGFR}_{\text {Exam } 1}-\mathrm{eGFR}_{\text {Exam } 3}\right) /($ number of days between Exam 1 and Exam 3).

\section{Statistical analysis}

We used two-sample t-tests for continuous variables or chi-squared tests for categorical variables to compare sociodemographic and clinical characteristics of study participants in our analytic cohort to those excluded for missing ISEL data. We used the same statistical tests to compare sociodemographic and clinical characteristics of study participants with low vs. high functional social support as defined by our primary exposure variable, ISEL. Because these tests were not part of our primary aims and hypotheses, we did not adjust for multiple testing. To evaluate the association of social support based on both ISEL or social network size with our outcomes of interest, we evaluated each social support measure in separate models as either continuous (ISEL score or social network size) or categorical variables (low vs. high social support). Multivariable logistic regression models used to determine the relationship between social support and prevalent CKD were adjusted for age, sex, income, education, BMI, smoking status, diabetes, and hypertension. Multivariable linear regression models to determine the relationship between social support and eGFR decline were additionally adjusted for baseline ACR and eGFR. We added an interaction term [ISEL (or social network size) $\times$ age] to examine if age moderated the effect of social support on eGFR decline.

In secondary analyses, we stratified our models by marital status as marital status can have a significant role in social support but is not measured in either ISEL or social network size [5]. Recognizing the measurement bias of determining annual change in kidney function decline with only two timepoints, we added a secondary outcome, rapid renal function decline (RRFD). We defined RRFD as a $>30 \%$ decline in eGFR between Exams 1 and 3, an approximately ten-year period. Multivariable logistic regression models used to determine the relationship between social support and RRFD had the same adjustment variables as the models for eGFR decline. To identify associations with specific aspects of social support, we also evaluated each social support domain (for social network size, individual questions) as the exposure variables. All analyses were performed with SAS Version 9.4 (SAS Institute, Cary NC) and R 3.4.0 (R Core Team, Vienna Austria) and used a two-sided 0.05 level for statistical significance.

\section{Results \\ Cohort characteristics}

Of 5301 JHS participants, 4015 (76\%) completed the ISEL at baseline. We excluded 1344 participants because of missing kidney function measures or covariates at baseline, leaving 2671 in our analytic cohort for the prevalent CKD outcome model. Similarly, we identified 2589 participants with diagnoses of diabetes, hypertension or CKD at baseline, but excluded 1345 participants because of missing kidney function measures and/or covariates, leaving 1244 in our analytic cohort for the kidney function decline outcome models (eGFR decline and RRFD) (Fig. 1). Compared to JHS participants who did not complete ISEL at baseline, those who did complete ISEL were more likely to have high school 


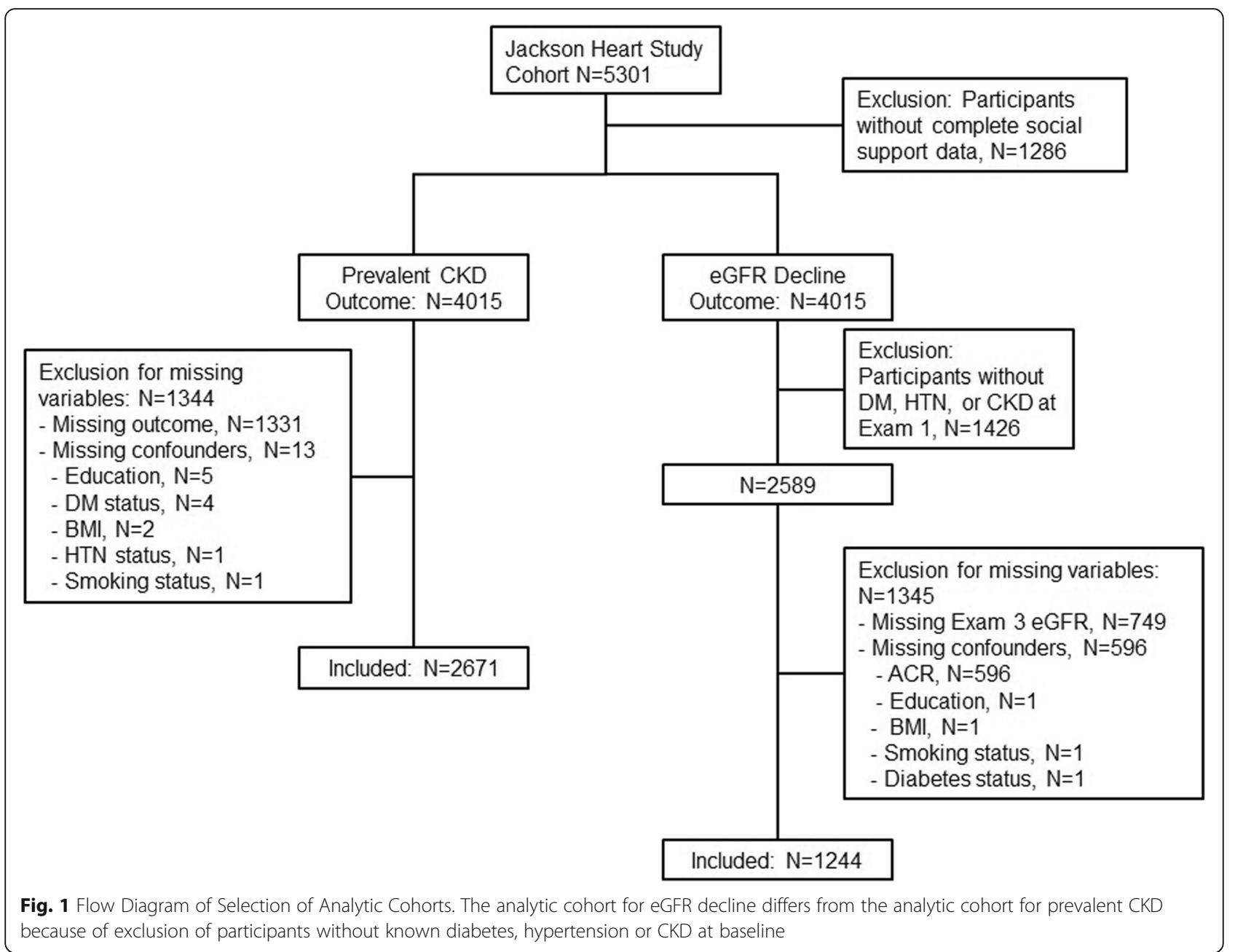

education (64\% vs. $52 \%)$ and income above poverty level (53\% vs. $43 \%$ ), and less likely to have hypertension (59\% vs. $63 \%)$, diabetes ( $21 \%$ vs. $24 \%)$, and CKD ( $12 \%$ vs. $15 \%)$. (Table 1). Characteristics of participants with and without CKD are shown in Additional file 1: Table S1.

\section{Social support}

The average ISEL score was relatively high, $37.0(\mathrm{SD}=7.2)$, and $21.0 \%(n=843)$ of participants reported low functional social support (ISEL < 32). Among those with low functional social support, $85.3 \%(n=719)$ also had low structural social support (social network size $<8$ ). Participants with low functional social support (vs. higher) were more likely to have lower income ( $47 \%$ vs. $28 \%$ ), be current or former tobacco users (39\% vs. $30 \%)$, and to have diabetes (25\% vs. $21 \%$ ) (Table 2 ).

\section{Functional social support and kidney function}

Prevalent CKD was more common among participants with low (vs. high) functional social support (22\% vs $17 \%$ ). However, among the 2589 participants with diabetes, hypertension or CKD at baseline, there was no difference in annual rate of eGFR decline between participants with low vs. high functional social support $(1.3 \pm 2.2 \mathrm{ml} / \mathrm{min} /$ $1.73 \mathrm{~m}^{2}$ vs. $\left.1.4 \pm 2.1 \mathrm{ml} / \mathrm{min} / 1.73 \mathrm{~m}^{2}\right)$. In multivariable regression modeling, we found no association between functional social support (total ISEL score) and prevalent CKD (OR $0.99,95 \%$ CI $0.98,1.01)$ or eGFR decline $(\beta=-0.01$, $95 \%$ CI $-0.02,0.01$ ) (Table 3). These findings were unchanged in models with dichotomized ISEL as the exposure variable: prevalent CKD (OR 1.14, 95\% CI 0.88, 1.48) and eGFR decline ( $\beta=0,95 \%$ CI $-0.28,0.28)$. Age did not modify the relationship between functional social support and eGFR decline $(p=0.71)$. Of the models with each ISEL subscale as exposure variables; we found the ISEL self-esteem domain score was associated with prevalent CKD (OR 0.94, 95\% CI 0.89,0.99), and the ISEL appraisal and tangible domain scores had significant but modest associations with eGFR decline $(\beta=-0.07,95 \% \mathrm{CI}-0.13,0)$ and $(\beta=0.07,95 \%$ CI $0.01,0.13)$, respectively (Table 3 ). Total ISEL score was not associated with prevalent CKD (OR 1.00, 95\% CI 0.98, 1.02) or eGFR decline 
Table 1 Comparison JHS Participants Included and Excluded in Analyses

\begin{tabular}{|c|c|c|c|c|}
\hline & Overall $(n=5301)$ & Included $(n=4015)$ & Excluded $(n=1286)$ & $P$-value \\
\hline \multicolumn{5}{|l|}{ Demographics } \\
\hline Age, year & $55.4 \pm 12.8$ & $54.8 \pm 12.6$ & $57.2 \pm 13.6$ & $<0.01$ \\
\hline Men & $1934(36.5)$ & $1436(35.8)$ & $498(38.7)$ & 0.06 \\
\hline High school education & $3232(61)$ & $2567(63.9)$ & $665(51.7)$ & $<0.01$ \\
\hline Income & & & & $<0.01$ \\
\hline$\leq 1.5$ times the poverty level & $1798(33.9)$ & $1297(32.3)$ & $501(39)$ & \\
\hline$>1.5$ times the poverty level & $2683(50.6)$ & $2130(53.1)$ & $553(43)$ & \\
\hline Missing & $820(15.5)$ & $588(14.6)$ & $232(18)$ & \\
\hline \multicolumn{5}{|l|}{ Renal Disease Risk Factors } \\
\hline Body Mass Index & $31.8 \pm 7.2$ & $31.8 \pm 7.2$ & $31.6 \pm 7.3$ & 0.52 \\
\hline Smoking status & & & & 0.07 \\
\hline Never & $3574(67.4)$ & $2738(68.2)$ & $836(65)$ & \\
\hline Former or current & $1716(32.4)$ & $1275(31.8)$ & $441(34.3)$ & \\
\hline Hypertension ( $n=5278)$ & $3169(60.0)$ & $2365(58.9)$ & $804(62.5)$ & $<0.01$ \\
\hline Diabetes $(n=5240)$ & $1152(21.9)$ & $845(21.0)$ & $307(23.9)$ & 0.01 \\
\hline \multicolumn{5}{|l|}{ Renal Function } \\
\hline Annual eGFR decline & $1.3 \pm 2$ & $1.2 \pm 2$ & $1.4 \pm 2$ & 0.09 \\
\hline eGFR at Visit 1 & $94.2 \pm 22$ & $94.9 \pm 21.5$ & $91.7 \pm 23.3$ & $<0.01$ \\
\hline $\log A C R$ at Visit 1 & $2.2 \pm 1.3$ & $2.1 \pm 1.2$ & $2.3 \pm 1.3$ & $<0.01$ \\
\hline Albuminuria at Visit 1 & & & & 0.16 \\
\hline No & $2875(54.2)$ & $2287(57)$ & $588(45.7)$ & \\
\hline Yes & $429(8.1)$ & $328(8.2)$ & $101(7.9)$ & \\
\hline Missing & $1997(37.7)$ & $1400(34.9)$ & $597(46.4)$ & \\
\hline CKD at Visit 1 & & & & $<0.01$ \\
\hline No & $2749(51.9)$ & $2197(54.7)$ & $552(42.9)$ & \\
\hline Yes & $673(12.7)$ & $487(12.1)$ & $186(14.5)$ & \\
\hline Missing & $1879(35.4)$ & $1331(33.2)$ & $548(42.6)$ & \\
\hline \multicolumn{5}{|l|}{ Social Support } \\
\hline Functional (ISEL score) & $37 \pm 7.2$ & $37 \pm 7.2$ & $35.8 \pm 7.1$ & 0.30 \\
\hline Structural (Social network size) & $5.9 \pm 2.7$ & $5.9 \pm 2.7$ & $6 \pm 2.7$ & 0.28 \\
\hline
\end{tabular}

Data expressed as $n$ (\%) or mean \pm SD

$(\mathrm{B}=0,95 \% \mathrm{CI}-0.02,0.02)$ in models stratified by marital status or with RRFD (OR 1.00, 95\% CI 0.98,1.02).

\section{Structural social support and kidney function}

In analyses of our secondary exposure variable, social network size, there was no association with prevalent CKD (OR $0.99,95 \%$ CI $0.95,1.03)$ or eGFR decline $(\beta=0.00$, $95 \%$ CI $-0.04,0.04)$. (Table 3 ). These findings were unchanged in models with dichotomized social network size as the exposure variable: prevalent CKD (OR 1.06, 95\% CI $0.84,1.34)$ and eGFR decline $(\beta=-0.02,95 \%$ CI $-0.25,0$. $22)$. Age did not modify the relationship between structural social support and eGFR decline $(p=0.72)$. Of models with individual social network items as the exposure variables, we found no association between individual social network items and prevalent CKD or eGFR decline. Social network size was not associated with prevalent CKD (OR 1.00, 95\% CI 0.94, 1.06) or eGFR decline $(\beta=$ $0.01,95 \%$ CI $-0.05,0.07)$ in models stratified by marital status or with RRFD (OR 1.00, 95\% CI 0.95,1.06).

\section{Discussion}

In this cohort of African American adults, we found that participants with low functional and/or structural social support were not more likely to have CKD. Additionally, among participants with CKD or at high risk due to diabetes and hypertension, high levels of functional and structural social support were not associated with faster decline in kidney function. However, within individual domains, higher ISEL self-esteem domain score appeared to 
Table 2 Baseline Characteristics of analytic sample by level of social support

\begin{tabular}{|c|c|c|c|c|}
\hline Variable & Total $(N=4015)$ & $\begin{array}{l}\text { Low Social Support } \\
\text { ISEL < } 32(N=843)\end{array}$ & $\begin{array}{l}\text { High Social Support } \\
\text { ISEL } \geq 32(N=3172)\end{array}$ & $P$-value \\
\hline \multicolumn{5}{|l|}{ Demographics } \\
\hline Age & $54.8 \pm 12.6$ & $54.8 \pm 13.2$ & $54.8 \pm 12.4$ & 0.87 \\
\hline Men & $1436(35.8)$ & $307(36.4)$ & 1129 (35.6) & 0.69 \\
\hline High school education & $2567(63.9)$ & $430(51)$ & $2137(67.4)$ & $<0.01$ \\
\hline Income & & & & $<0.01$ \\
\hline$\leq 1.5$ times the poverty level & $1297(32.3)$ & $400(47.4)$ & $897(28.3)$ & \\
\hline$>1.5$ times the poverty level & $2130(53.1)$ & $319(37.8)$ & $1811(57.1)$ & \\
\hline Missing & $588(14.6)$ & $124(14.7)$ & $464(14.6)$ & \\
\hline \multicolumn{5}{|l|}{ Renal Disease Risk Factors } \\
\hline Body Mass Index $(n=4008)$ & $31.8 \pm 7.2$ & $32.5 \pm 7.6$ & $31.6 \pm 7.1$ & $<0.01$ \\
\hline Smoking status & & & & $<0.01$ \\
\hline Never & $2738(68.2)$ & $518(61.4)$ & $2220(70)$ & \\
\hline Former or current & $1275(31.8)$ & 325 (38.6) & $950(29.9)$ & \\
\hline Missing & $2(0)$ & $0(0)$ & $2(0.1)$ & \\
\hline Hypertension & & & & 0.11 \\
\hline No HTN & $1631(40.6)$ & $322(38.2)$ & $1309(41.3)$ & \\
\hline Controlled HTN & $1198(29.8)$ & $254(30.1)$ & $944(29.8)$ & \\
\hline Uncontrolled HTN & $1167(29.1)$ & $262(31.1)$ & $905(28.5)$ & \\
\hline Have HTN but can't determine control ${ }^{a}$ & $16(0.4)$ & $5(0.6)$ & $11(0.3)$ & \\
\hline Missing & $3(0.1)$ & $0(0)$ & $3(0.1)$ & \\
\hline Diabetes & & & & 0.03 \\
\hline No DM & $3129(77.9)$ & $633(75.1)$ & $2496(78.7)$ & \\
\hline Controlled DM & $422(10.5)$ & $107(12.7)$ & $315(9.9)$ & \\
\hline Uncontrolled DM & $423(10.5)$ & $98(11.6)$ & $325(10.2)$ & \\
\hline Missing & $41(1)$ & $5(0.6)$ & $36(1.1)$ & \\
\hline \multicolumn{5}{|l|}{ Renal Function } \\
\hline Annual renal function decline $(n=2947)$ & $1.2 \pm 2$ & $1.3 \pm 2$ & $1.2 \pm 2$ & 0.8 \\
\hline eGFR at Visit $1(n=3953)$ & $94.9 \pm 21.5$ & $94.5 \pm 24.2$ & $95.1 \pm 20.7$ & 0.51 \\
\hline $\log A C R$ at Visit $1(n=2614)$ & $2.1 \pm 1.2$ & $2.2 \pm 1.3$ & $2.1 \pm 1.2$ & 0.30 \\
\hline Albuminuria at Visit 1 & & & & 0.15 \\
\hline No & $2287(57)$ & $442(52.4)$ & $1845(58.2)$ & \\
\hline Yes & $328(8.2)$ & $75(8.9)$ & $253(8)$ & \\
\hline Missing & $1400(34.9)$ & $326(38.7)$ & $1074(33.9)$ & \\
\hline CKD at Visit 1 & & & & 0.02 \\
\hline No & $2197(54.7)$ & $423(50.2)$ & $1774(55.9)$ & \\
\hline Yes & $487(12.1)$ & $118(14)$ & $369(11.6)$ & \\
\hline Missing & $1331(33.2)$ & $302(35.8)$ & 1029 (32.4) & \\
\hline \multicolumn{5}{|l|}{ Social Support } \\
\hline Functional (ISEL score) & $37 \pm 7.2$ & $26.1 \pm 4.7$ & $39.9 \pm 4.4$ & - \\
\hline Structural (Social network size) & $5.88 \pm 2.67$ & $4.81 \pm 2.45$ & $6.17 \pm 2.65$ & $<0.01$ \\
\hline
\end{tabular}

Data expressed as $n$ (\%) or mean \pm SD based on total of 4015 participants, unless otherwise specified

annable to identify if achieve Hypertension control in participants with missing diabetes status and BP between 130/80 and 140/90 
Table 3 Adjusted Analyses of Social Support and Kidney Function

\begin{tabular}{ccc}
\hline Variable & $\begin{array}{l}\text { Relative Odds of Prevalent } \\
\text { CKD }^{\mathrm{a}} \text { OR }(95 \% \mathrm{Cl})\end{array}$ & $\begin{array}{l}\text { Difference in eGFR } \\
\text { decline }^{\mathrm{b}} \beta(95 \% \mathrm{Cl})\end{array}$ \\
\hline $\begin{array}{l}\text { Functional Social Support } \\
\text { ISEL Total }\end{array}$ & $0.99(0.98,1.01)$ & $-0.01(-0.02,0.01)$ \\
ISEL Subscales & & \\
Appraisal & $1.03(0.96,1.10)$ & $\mathbf{- 0 . 0 7}(-\mathbf{0 . 1 3}, \mathbf{0 . 0 0})$ \\
Belonging & $0.99(0.93,1.05)$ & $-0.02(-0.09,0.04)$ \\
Self-esteem & $\mathbf{0 . 9 4}(\mathbf{0 . 8 9 , 0 . 9 9 )}$ & $-0.02(-0.08,0.04)$ \\
Tangible & $1.01(0.95,1.07)$ & $\mathbf{0 . 0 7}(\mathbf{0 . 0 1}, \mathbf{0 . 1 3})$
\end{tabular}

Structural Social Support

Social network $0.99(0.95,1.03) \quad 0(-0.04,0.04)$
size score ${ }^{d}$

Table reflects effect estimates of separate multivariable regression models using ISEL total score, ISEL subscales, and social network size score as exposure variables of interest. Data expressed as effect estimate [odds ratio (OR) or beta coefficient] and $95 \%$ confidence interval (Cl). Significant effect estimates $(p<.05)$ indicated in bold

${ }^{a}$ Model 1 has prevalent CKD as the outcome. Analyses conducted on complete cases, $n=2671$, and were adjusted for age, gender, income, education, body mass index, smoking status, diabetes, and hypertension

${ }^{b}$ Model 2 has eGFR decline in $\mathrm{ml} / \mathrm{min} / 1.73 \mathrm{~m}^{2}$ per year as the outcome. Analyses conducted on complete cases, $n=1244$ (participants with diabetes, hypertension or CKD at baseline), and were adjusted for age, gender, income, education, body mass index, smoking status, diabetes, and hypertension, albumin/creatinine ratio, and baseline eGFR

${ }^{c}$ Interpersonal Support Evaluation List (ISEL) is a validated measure of functional social support

${ }^{\mathrm{d}}$ Social network size is a measure of structural social support based on number and frequency of close contacts

be associated with lower odds of prevalent CKD. Although modest, this finding suggests that further study into the role of self-esteem in CKD outcomes could uncover novel interventions targeting CKD among African Americans.

To our knowledge, this is the first study to examine the extent to which social support is independently associated with CKD outcomes in an African American cohort. Two observational studies of patients receiving dialysis demonstrate high social support was independently associated with improved survival $[7,10]$. One cross-sectional study in African Americans identified high social support to be independently associated with modest reduction in odds of diabetes and hypertension [18]. A separate study conducted in African Americans demonstrated that increasing social support was associated with more self-management behavior (e.g., adding more fruits and vegetables to meal plan or counting number of fruits or vegetables eaten per day) and subsequently more fruit and vegetable consumption [17]. In contrast to those studies, we did not identify an independent relationship between functional and structural measures of social support and CKD outcomes. However, our study adds to these findings by demonstrating that high self-esteem, as it relates to social support, has a modest independent association with lower odds of prevalent CKD. The associations between the tangible and appraisal domains of functional social support and kidney function decline were too modest to represent a clinically meaningful change in eGFR.

Our findings may be explained, in part, by our study design. Although functional and structural social support have been associated with some clinical outcomes, it may have been more appropriate to measure the association between CKD and specific self-management behaviors. Self-management behaviors, such as physical activity or adherence to diabetes and hypertension medications, could be in the causal pathway between social support and CKD outcomes [28]. Thus, additional steps to identify an association between social support and self-management behaviors is warranted. Our findings may also be influenced by the low prevalence of CKD (13\%) and low social support (21\%) in the JHS. Most importantly, our findings may be explained by the appropriateness of our exposure variables, the ISEL and social network measures, in this African American cohort. The ISEL used in our study has been administered in other studies with African American participants, with similar mean total score (ranging from 30 to 37) [19, 27]. However, the survey's items may be subject to measurement bias. Most participants reported high scores on ISEL items which may represent social desirability bias, or response bias that occurs when respondents want to give responses that are socially acceptable [29]. Alternatively, the ISEL items may not account for the cultural nuances that tend to shape how African Americans may perceive their level of social support [9]. Our assessment of social network size was limited in its ability to discretely assess additional aspects of structural social support (e.g., church membership, social group involvement) that are captured in the Berkman social network index [5, 6]. Overall, these findings, along with evidence from other studies examining psychosocial factors in African Americans, endorse additional research on the content validity of psychosocial instruments in this population.

We did find that higher scores on the ISEL selfesteem domain were associated with a modest reduction in odds of prevalent CKD. The ISEL self-esteem domain assesses an individual's ability to have a positive comparison when comparing oneself with others, and it is correlated with the widely used Rosenberg self-esteem scale [30]. It is plausible that individuals who have higher perceived self-esteem social support are less likely to have CKD in settings where this high self-esteem is tied with high self-efficacy. Individuals with high self-efficacy are more likely to engage in health behaviors and lifestyle modifications that can prevent onset and/or progression of CKD [31]. If self-esteem from social support is truly a buffer against development of CKD, we would expect that the self-esteem score would also be associated 
with a slower decline in eGFR. Absence of that finding in this study does not rule out the possibility that some individuals with risk factors for CKD, like diabetes or hypertension, may possess some level of psychosocial resilience that buffers against the onset of CKD. A longitudinal cohort study that evaluates changes in both social support and health status over time may uncover a temporal relationship between self-esteem social support and kidney function decline. More evidence supporting this relationship could increase the argument for behavioral interventions that aim to enhance self-esteem social support in African Americans with risk factors for CKD.

The important strengths of this study are its: 1) large African American community-based cohort, 2) rich psychosocial assessment data to assess both functional and structural social support in CKD, and 3) longitudinal measures of kidney function. Despite these strengths, our study has its limitations. First, the cross-sectional study design limits our ability to make any causal or temporal inference about the association of social support, specifically self-esteem, with prevalent CKD. For models of eGFR decline, we restricted the analytic cohort to participants with baseline CKD, diabetes, or hypertension. While those comorbidities are important risk factors for eGFR decline, these comorbidities may have concealed an association of social support with eGFR decline. Also, we used social support measures that have not been formally validated to confirm the best cutoff for low social support. Despite this limitation, these variables as continuous measures did not demonstrate an association with kidney function outcomes. We were unable to account for unmeasured confounders, such as changes in health status and social support over time. It is plausible that lack of association in those models may be explained, in part, by an increase in perceived social support in the time between research visits and/or limited sample size. Last, we examined data from research volunteers so these findings have limited generalizability to all African Americans.

\section{Conclusion}

In an African American community-based cohort study, functional or structural social support measures were not independently associated with CKD outcomes. However, self-esteem measures showed a modest independent association with prevalent CKD. Because of strong evidence underlying the role of psychosocial factors in chronic disease self-management, there remains a need for additional research on the type and extent of social support that may be modified to improve kidney function outcomes in African Americans.

\section{Additional file}

Additional file 1: Table S1. Comparison of characteristics of JHS participants with and without CKD. (DOCX $19 \mathrm{~kb}$ )

\section{Abbreviations}

ACR: Albumin-to-creatinine ration; Cl: Confidence interval; CKD: Chronic kidney disease; EGFR: Estimated glomerular filtration rate; ISEL: Interpersonal Support Evaluation List; JHS: Jackson Heart Study; OR: Odds ratio;

RRFD: Rapid renal function decline

\section{Acknowledgements}

The authors wish to thank the staffs and participants of the JHS. The findings reported here were presented in poster format at the 2018 American Society of Nephrology Kidney Week in San Diego, CA.

\section{Authors' contributions}

$\mathrm{RH}$ led study conceptualization, analysis plan development, interpretation of results, and manuscript preparation. CAD performed primary data analysis.

MS, CCE, TW, JR, JP, NB, JS, CT, WW, YM, BY, LEB, and CD all contributed meaningfully to research analysis plan development, interpretation of study results, and manuscript preparation. All authors read and approved the final manuscript.

\section{Funding}

The Jackson Heart Study (JHS) is supported and conducted in collaboration with Jackson State University (HHSN268201800013I), Tougaloo College (HHSN268201800014I), the Mississippi State Department of Health (HHSN268201800015I) and the University of Mississippi Medical Center (HHSN268201800010l, HHSN268201800011I and HHSN268201800012I) contracts from the National Heart, Lung, and Blood Institute (NHLBI) and the National Institute for Minority Health and Health Disparities (NIMHD). RH is supported by the National Institute on Aging (K76AG059930-01). CAD is supported by the Duke Clinical and Translational Science Award

(UL1TR001117). BY is supported in part by funding from the Veterans Affairs Puget Sound Health Care System. The funding sources had no role in the design of the study and collection, analysis, and interpretation of data and in writing the manuscript. The views expressed in this manuscript are those of the authors and do not necessarily represent the views of the National Heart, Lung, and Blood Institute; the National Institutes of Health; the U.S. Department of Health and Human Services or Department of Veterans Affairs.

Availability of data and materials

The data that support the findings of this study are available from The Jackson Heart Study but restrictions apply to the availability of these data, which were used under license for the current study, and so are not publicly available. Data are however available from the authors upon reasonable request and with permission of The Jackson Heart Study.

Ethics approval and consent to participate

The JHS protocol was approved by the Institutional Review Boards at Mississippi Medical Center, Jackson State University, and Tougaloo College, and all participants provided written informed consent.

Consent for publication

Not applicable.

\section{Competing interests}

The authors declare that they have no competing interests.

\section{Author details}

${ }^{1}$ Duke University, Durham, NC, USA. ${ }^{2}$ University of Mississippi Medical Center, Jackson, MS, USA. ${ }^{3}$ University of Georgia, Athens, GA, USA. ${ }^{4}$ University of Washington, Seattle, WA, USA. 
Received: 1 March 2019 Accepted: 23 June 2019

Published online: 15 July 2019

\section{References}

1. Barbour SJ, Schachter M, Er L, Djurdjev O, Levin A. A systematic review of ethnic differences in the rate of renal progression in CKD patients. Nephrol Dial Transplant. 2010;25(8):2422-30.

2. Tarver-Carr ME, Powe NR, Eberhardt MS, LaVeist TA, Kington RS, Coresh J, et al. Excess risk of chronic kidney disease among African-American versus white subjects in the United States: a population-based study of potential explanatory factors. J Am Soc Nephrol. 2002;13(9):2363-70.

3. Cohen SD, Sharma T, Acquaviva K, Peterson RA, Patel SS, Kimmel PL. Social support and chronic kidney disease: an update. Adv Chronic Kidney Dis. 2007;14(4):335-44

4. Tay L, Tan K, Diener E, Gonzalez E. Social relations, health behaviors, and health outcomes: a survey and synthesis. Appl Psychol Health Well Being. 2013;5(1):28-78.

5. Berkman LF, Syme SL. Social networks, host resistance, and mortality: a nineyear follow-up study of Alameda County residents. Am J Epidemiol. 1979; 109(2):186-204

6. Berkman LF, Vaccarino V, Seeman T. Gender differences in cardiovascular morbidity and mortality: the contribution of social networks and support. Ann Behav Med. 1993;15(2-3):112-8.

7. Kimmel PL, Peterson RA, Weihs KL, Simmens SJ, Alleyne S, Cruz I, et al. Psychosocial factors, behavioral compliance and survival in urban hemodialysis patients. Kidney Int. 1998:54(1):245-54.

8. Nagayoshi M, Everson-Rose SA, Iso H, Mosley TH Jr, Rose KM, Lutsey PL. Social network, social support, and risk of incident stroke: Atherosclerosis Risk in Communities study. Stroke. 2014;45(10):2868-73.

9. Sims RC, Levy SA, Mwendwa DT, Callender CO, Campbell AL Jr. The influence of functional social support on executive functioning in middleaged African Americans. Neuropsychol Dev Cogn B Aging Neuropsychol Cogn. 2011;18(4):414-31.

10. Untas A, Thumma J, Rascle N, Rayner H, Mapes D, Lopes AA, et al. The associations of social support and other psychosocial factors with mortality and quality of life in the dialysis outcomes and practice patterns study. Clin J Am Soc Nephrol. 2011:6(1):142-52.

11. Gallant MP. The influence of social support on chronic illness selfmanagement: a review and directions for research. Health Educ Behav. 2003;30(2):170-95

12. Uchino BN. Social support and health: a review of physiological processes potentially underlying links to disease outcomes. J Behav Med. 2006;29(4):377-87.

13. Ajrouch KJ, Reisine S, Lim S, Sohn W, Ismail A. Perceived everyday discrimination and psychological distress: does social support matter? Ethn Health. 2010;15(4):417-34.

14. Ford ME, Tilley BC, McDonald PE. Social support among African-American adults with diabetes, part 2: a review. J Natl Med Assoc. 1998;90(7):425-32.

15. Coulon SM, Wilson DK. Social support buffering of the relation between low income and elevated blood pressure in at-risk African-American adults. J Behav Med. 2015;38(5):830-4

16. Cohen S, Hoberman HM. Positive Events and Social Supports as Buffers of Life Change Stress1. 1983;13(2):99-125.

17. O'Neal CW, Wickrama KA, Ralston PA, Ilich JZ, Harris CM, Coccia C, et al. Examining change in social support and fruit and vegetable consumption in African American adults. J Nutr Health Aging. 2014;18(1):10-4.

18. Hernandez DC, Reitzel LR, Wetter DW, McNeill LH. Social support and cardiovascular risk factors among black adults. Ethn Dis. 2014;24(4):444-50.

19. Porter A, Fischer MJ, Brooks D, Bruce M, Charleston J, Cleveland WH, et al. Quality of life and psychosocial factors in African Americans with hypertensive chronic kidney disease. Transl Res. 2012;159(1):4-11.

20. Nicholson NR. A review of social isolation: an important but underassessed condition in older adults. J Prim Prev. 2012;33(2-3):137-52.

21. Liddy C, Blazkho V, Mill K. Challenges of self-management when living with multiple chronic conditions: systematic review of the qualitative literature. Can Fam Physician. 2014;60(12):1123-33.

22. White AM, Philogene GS, Fine L, Sinha S. Social support and self-reported health status of older adults in the United States. Am J Public Health. 2009: 99(10):1872-8

23. Taylor HA Jr, Wilson JG, Jones DW, Sarpong DF, Srinivasan A, Garrison RJ et al. Toward resolution of cardiovascular health disparities in African
Americans: design and methods of the Jackson Heart Study. Ethn Dis. 2005; 15(4 Suppl 6):S6-4-17.

24. Taylor HA Jr. The Jackson heart study: an overview. Ethn Dis. 2005;15(4 Suppl 6):S6 -1-3.

25. Fuqua SR, Wyatt SB, Andrew ME, Sarpong DF, Henderson FR, Cunningham $M F$, et al. Recruiting African-American research participation in the Jackson heart study: methods, response rates, and sample description. Ethn Dis. 2005;15(4 Suppl 6):S6-18-29.

26. Cohen S, Mermelstein R, Kamarck T, Hoberman HM. Measuring the functional components of social support. In: Sarason IG, Sarason BR, editors. Social support: theory, Research and applications NATO ASI series (D: Behavioural and social sciences), vol. 24. Dordrecht: Springer; 1985.

27. Payne TJ, Andrew M, Butler KR, Wyatt SB, Dubbert PM, Mosley TH. Psychometric evaluation of the interpersonal support evaluation list-short form in the ARIC study cohort. SAGE Open. 2012;2(3):2158244012461923.

28. Schrauben SJ, Hsu JY, Rosas SE, Jaar BG, Zhang X, Deo R, et al. CKD selfmanagement: phenotypes and associations with clinical outcomes. Am J Kidney Dis. 2018;72(3):360-70.

29. Althubaiti A. Information bias in health research: definition, pitfalls, and adjustment methods. J Multidiscip Healthc. 2016;9:211-7.

30. Rosenberg M. Society and the adolescent self-image. Princeton, NJ: Princeton University Press; 1965.

31. Curtin RB, Walters BA, Schatell D, Pennell P, Wise M, Klicko K. Self-efficacy and self-management behaviors in patients with chronic kidney disease. Adv Chronic Kidney Dis. 2008;15(2):191-205

\section{Publisher's Note}

Springer Nature remains neutral with regard to jurisdictional claims in published maps and institutional affiliations.
Ready to submit your research? Choose BMC and benefit from:

- fast, convenient online submission

- thorough peer review by experienced researchers in your field

- rapid publication on acceptance

- support for research data, including large and complex data types

- gold Open Access which fosters wider collaboration and increased citations

- maximum visibility for your research: over $100 \mathrm{M}$ website views per year

At $\mathrm{BMC}$, research is always in progress.

Learn more biomedcentral.com/submissions 